\title{
Vitreous levels of intercellular adhesion molecule 1 (ICAM-1) as a risk indicator of proliferative vitreoretinopathy
}

\author{
G Astrid Limb, Anthony H Chignell
}

\begin{abstract}
Aim-To investigate whether high vitreous levels of the soluble intercellular adhesion molecule 1 (sICAM-1) may be related to clinical risk factors of proliferative vitreoretinopathy (PVR) and whether their measurement may serve as an additional risk indicator of this complication in eyes with rhegmatogenous retinal detachment (RRD).
\end{abstract}

Methods-Levels of sICAM-1 were measured by enzyme linked immunosorbent assays (ELISA) in vitreous from 36 eyes with RRD clinically considered to be at high risk of developing PVR (large retinal breaks, vitreous haemorrhage, long standing RRD, and previous vitreoretinal surgery). Levels of sICAM-1 in this group were compared with those in vitreous from 31 eyes with RRD without clinical risk factors for PVR, 32 eyes with established PVR and 10 eyes with macular holes.

Results-Vitreous from eyes with RRD at high risk of developing PVR contained significantly higher levels of SICAM-1 (range 6.1-97.7 ng/ml; Mann-Whitney test, $p=0.0002$ ) than those from eyes with RRD at low risk of developing this complication (range 4.8-17.7 $\mathrm{ng} / \mathrm{ml}$ ). Vitreous sICAM-1 levels in eyes with RRD at high risk of developing PVR were significantly lower than in eyes with established PVR $(p=0.037)$, but higher than in eyes with macular holes $(p<0.0001)$. Levels of sICAM-1 $\geqslant 15 \mathrm{ng} / \mathrm{ml}(3 \times$ median of the levels present in control eyes) provide a useful cut off point for a highly specific test $(96.7 \%)$ with high positive $(91.6 \%)$ and negative $(96.7 \%)$ predictive values, despite a relatively low sensitivity $(30.5 \%)$.

Conclusions-The present findings suggest that laboratory measurement of sICAM-1 levels in vitreous from eyes with RRD may constitute an additional factor for identifying patients at high risk of PVR. Hence, determination of SICAM-1 levels may aid in the monitoring of patients likely to develop this complication and in the identification of patients who may benefit from adjuvant antiinflammatory therapy.

(Br F Ophthalmol 1999;83:953-956)
Despite recent advances in the understanding of the molecular mechanisms involved in the pathogenesis of proliferative vitreoretinopathy (PVR), the role of adjunctive therapy is at present uncertain and treatment relies upon vitreoretinal surgery. ${ }^{1}$ There is a general consensus that, in addition to surgical procedures, it may of value to administrate antiinflammatory therapy either as prophylaxis or as adjunct to improve the visual outcome and prevent the chronicity of this condition. ${ }^{23}$ However, owing to the potential toxicity of the antiproliferative drugs currently available, ${ }^{3-5}$ it may be justifiable to restrict the use of pharmacological therapy to those who are considered to be at special risk of developing PVR. Development of laboratory tests to identify those individuals who would be most likely to benefit from adjuvant therapy would therefore assist the vitreoretinal surgeon in his clinical and surgical management of rhegmatogenous retinal detachment (RRD).

Several inflammatory factors, including cytokines, are often observed in vitreous ${ }^{67}$ and retinal membranes from eyes with $\mathrm{PVR},{ }^{8}{ }^{9}$ but none of these molecules has been shown to serve as a risk indicator for the development of this complication. During initiation of the inflammatory process, cytokines and chemotactic factors locally released promote the migration of inflammatory cells to the injured site. This is due to upregulation in the expression of vascular endothelial cell adhesion molecules by these factors. ${ }^{10}$ Among these, the intercellular adhesion molecule 1 (ICAM-1) is crucial for leucocyte migration through endothelial cell junctions and into the site of inflammation. ${ }^{10}$ This molecule is expressed by vascular endothelial cells and retinal pigment epithelium (RPE) among other cells, ${ }^{11}{ }^{12}$ and may be present within the extracellular matrix of PVR membranes, ${ }^{13}$ and on the retinal endothelium of eyes with anterior PVR. ${ }^{14}$ During inflammation, adhesion molecules are shed from the cell surface, for which they can be found in soluble form in serum and body fluids. ${ }^{15}$ While physiological concentrations of soluble ICAM-1 (sICAM-1) are found in serum of healthy individuals, significantly raised levels of this molecule have been found in serum and inflammatory fluids from patients with various disorders ${ }^{15}$ and in vitreous
Accepted for publication 8 March 1999 
from patients with established PVR and other retinal proliferative disorders. ${ }^{16-18}$

Our previous observations that high levels of this molecule were present in two eyes which, at the time of vitrectomy for RRD, did not have PVR greater than grade $\mathrm{B}$, but that later developed this complication, ${ }^{17}$ prompted us to investigate whether high vitreous levels of sICAM-1 are related to the known clinical risk factors for PVR, and whether their laboratory measurement could be used as an additional factor to identify those patients likely to develop this complication. On this basis, we investigated the levels of sICAM-1 in vitreous from eyes with uncomplicated RRD with well recognised features of high clinical risk of developing PVR, ${ }^{19}{ }^{20}$ and compared these with the levels found in vitreous from eyes with RRD at low clinical risk of PVR, and eyes with established PVR. Vitreous samples from eyes undergoing pars plana vitrectomy for treatment of macular holes were used as controls.

\section{Patients and methods}

Vitreous and serum samples were obtained from 109 patients undergoing pars plana vitrectomy (PPV) for the treatment of uncomplicated RRD (less than grade B, 67 patients), established PVR (32 patients), or macular holes (10 patients). Of the 67 patients with uncomplicated RRD, 31 were at low clinical risk of PVR, while 36 were at high clinical risk of PVR, as judged by well recognised clinical features that predispose to this complication. ${ }^{1920}$ These included the presence of giant or very large retinal breaks (13 patients), severe vitreous haemorrhage (six patients), long standing RRD (>3 months) with history of previous retinal surgery (17 patients). In the uncomplicated RRD group, vitrectomy was considered the best choice of operation because of either opacities in the media (obscuring the fundal view), or to the complex size, site, or distribution of retinal breaks. Eyes with established PVR were grade $\mathrm{B}+$ according to the classification of Machemer et al. ${ }^{21}$ Ten vitreous specimens from eyes undergoing PPV for treatment of macular holes were used as disease controls. Clinical follow up of the patients under study revealed that none of the patients with low clinical risk of PVR developed this complication, while one of the 36 patients presenting with high clinical risk factors actually developed PVR. Vitreous specimens (approximately $0.75 \mathrm{ml}$ ) were centrifuged and transferred to cryotubes for storing at $-70^{\circ} \mathrm{C}$ until use. The study was approved by the local health authority ethics committee and it was performed in accordance with the ethical standards laid down in the 1964 Declaration of Helsinki.

Levels of SICAM-1 were measured by enzyme linked immunosorbent assays (ELISA) using commercially available kits (R\&D Systems, Abingdon) as follows. Microtitre well plates coated with anti-ICAM antibodies were incubated with $100 \mu \mathrm{l}$ of a 1:10 dilution of vitreous, together with $100 \mu \mathrm{l}$ of anti-ICAM-1HRP conjugate for 1.5 hours, after which antibodies and test samples were removed and the plate washed six times with phosphate buffered saline (PBS) containing $0.05 \%$ Tween-20. The amount of conjugated antibodies was detected by addition of $100 \mu \mathrm{l}$ of tetramethylbenzidine (substrate) and incubated for 30 minutes at room temperature. The reaction was stopped by addition of $100 \mu \mathrm{l}$ of $1 \mathrm{M} \mathrm{H}_{2} \mathrm{SO}_{4}$ and the absorbance read at $450 \mathrm{~nm}$, in a Dynatech MR5000 reader. Levels of sICAM-1 present in the vitreous or serum samples were interpolated from specific calibration curves prepared with known standard solutions. The specificity of the reaction was controlled by addition of interleukin 6 and human serum albumin to normal cadaveric vitreous to a final protein concentration of $3.0 \mathrm{mg} / \mathrm{ml}$ (similar to the levels observed in pathological specimens). Addition of these proteins did not modify the levels of sICAM-1 detected in the vitreous samples, thus indicating the specificity and validity of the test.

STATISTICAL ANALYSIS OF THE RESULTS

The significance of difference between corresponding groups of observations was evaluated by the Mann-Whitney test. Acceptable significance was recorded when $\mathrm{p}$ values were $<0.05$. The sensitivity, specificity, and positive and negative predictive values of SICAM-1 measurements were evaluated according to standard methods. ${ }^{22}$ For analysis, values corresponding to $3 \times$ and $2 \times$ the median levels of sICAM-1 observed in eyes with macular holes $(\geqslant 15$ $\mathrm{ng} / \mathrm{ml}$ and $\geqslant 10 \mathrm{ng} / \mathrm{ml}$, respectively) were taken as cut off points for evaluation of the test. A comparison was made between these levels and the presence or absence of clinical risk factors of PVR. Accordingly, sensitivity was defined as the prevalence of the positive test in subjects with clinical risk factors for PVR. Specificity was defined as the prevalence of the negative test in individuals without clinical risk factors for PVR. The positive predictive value was calculated as the proportion of subjects with a positive test who presented with clinical risk factors for PVR, while the negative predictive value was calculated as the proportion of subjects with negative test who did not present with clinical risk factor for PVR. ${ }^{22}$

\section{Results}

VITREOUS LEVELS OF SICAM-1 IN EYES WITH HIGH CLINICAL RISK OF PVR

Figure 1 shows that levels of sICAM-1 in vitreous from eyes with uncomplicated RRD at high clinical risk of PVR are significantly higher (range $6.1-97.8 \mathrm{ng} / \mathrm{ml}$ ) than in vitreous from eyes with RRD at low clinical risk of PVR (range 4.9-17.7 ng/ml: Mann-Whitney U test, $\mathrm{p}=0.00027)$ and vitreous from eyes with macular holes (range 4.8-7.6 ng/ml: MannWhitney $\mathrm{U}$ test, $\mathrm{p}=0.0000038$ ). However, vitreous sICAM-1 levels in eyes with RRD at high risk of developing PVR were significantly lower than in eyes with established PVR (Mann-Whitney U test: $p=0.037$ ), and vitreous levels of sICAM-1 in eyes with RRD at low risk of PVR, were significantly higher than in eyes with macular holes (Mann-Whitney U test: $p=0.00047)$. Only one of the 31 vitreous 


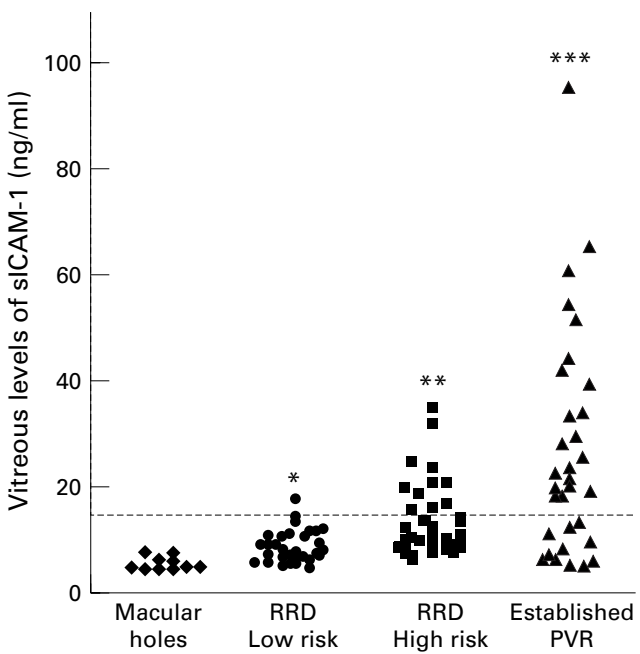

Figure 1 Levels of sICAM-1 in vitreous from eyes with rhegmatogenous retinal detachment (RRD) at high clinical risk of developing proliferative vitreoretinopathy (PVR). Comparison with vitreous levels of the same molecule in eyes with RRD at low risk of PVR, established PVR and eyes with macular holes. *Mann-Whitney $U$ test, $p=0.00047$ ( $v$ eyes with macular holes);

$\star \star M a n n-W h i t n e y U$ test), $p=0.00027$ ( $v$ RRD at low risk of PVR), $p=0.0000038$ ( $v$ eyes with macular holes); $\star * *$ Mann-Whitney $U$ test, $p=0.037$ ( $v$ RRD at high risk of PVR), $p=0.000075$ ( $v$ RRD at low risk of PVR). The broken line represents three times the median of the

ICAM-1 levels in vitreous from eyes with macular holes (15 $\mathrm{ng} / \mathrm{ml})$.

specimens from eyes without clinical risk factors for PDR contain more than $15 \mathrm{ng} / \mathrm{ml}$ sICAM-1. Analysis of sICAM-1 levels in patients with RRD showed that concentrations of $\geqslant 15 \mathrm{ng} / \mathrm{ml}$ ( $3 \times$ the median values observed in eyes with macular holes) taken as the cut off point for a positive test, provide a highly specific assay (96.7\%) with high positive $(91.6 \%)$ and negative $(96.7 \%)$ predictive values, despite a relatively low sensitivity $(30.5 \%)$ (Table 1$)$. Levels of sICAM-1 $\geqslant 10$ $\mathrm{ng} / \mathrm{ml}(2 \times$ the median values observed in eyes with macular holes) taken as the cut off point for a positive test, provide an increased sensitivity $(58.3 \%)$, although the specificity and positive and negative predictive values decreased considerably (67.7, 67.7, and $58.3 \%$, respectively) (Table 1 ).

LEVELS OF VITREOUS SICAM-1 IN RELATION TO CLINICAL RISK FACTORS FOR PVR

Analysis of vitreous specimens from individuals at high clinical risk of developing PVR showed that significantly high levels of sICAM-1 were present in vitreous from eyes with giant or large retinal breaks (range 6.11-31.9 ng/ml; Mann-Whitney $U$ test: $\mathrm{p}=0.0003$ ), eyes that had undergone previous operations (range 6.75-34.8 ng/ml; Mann-

Table 1 Analysis of the sensitivity, specificity, and predictive value of vitreous sICAM-1 measurement

\begin{tabular}{lllll}
\hline Factor for positive test & Sensitivityt & Specificity $\neq$ & $\begin{array}{l}\text { Positive predictive } \\
\text { valueS }\end{array}$ & $\begin{array}{l}\text { Negative predictive } \\
\text { value }\end{array}$ \\
\hline$\geqslant 15 \mathrm{ng} / \mathrm{ml}(3 \times \mathrm{MoC})$ & $11 / 36(30.5 \%)$ & $30 / 31(96.7 \%)$ & $11 / 12(91.6 \%)$ & $30 / 31(96.7 \%)$ \\
$\geqslant 10 \mathrm{ng} / \mathrm{ml}(2 \times \mathrm{MoC})$ & $21 / 36(58.3 \%)$ & $21 / 31(67.7 \%)$ & $21 / 31(67.7 \%)$ & $21 / 36(58.3 \%)$
\end{tabular}

${ }^{\star} \mathrm{MoC}$, median of the control; tprevalence of positive test in subjects with clinical risk factors of PVR, łprevalence of negative test in subjects without clinical risk factors of PVR, Sproportion of subjects with positive tests that presented with clinical risk of PVR, Iproportion of subjects with negative test that did not present with clinical risk of PVR.

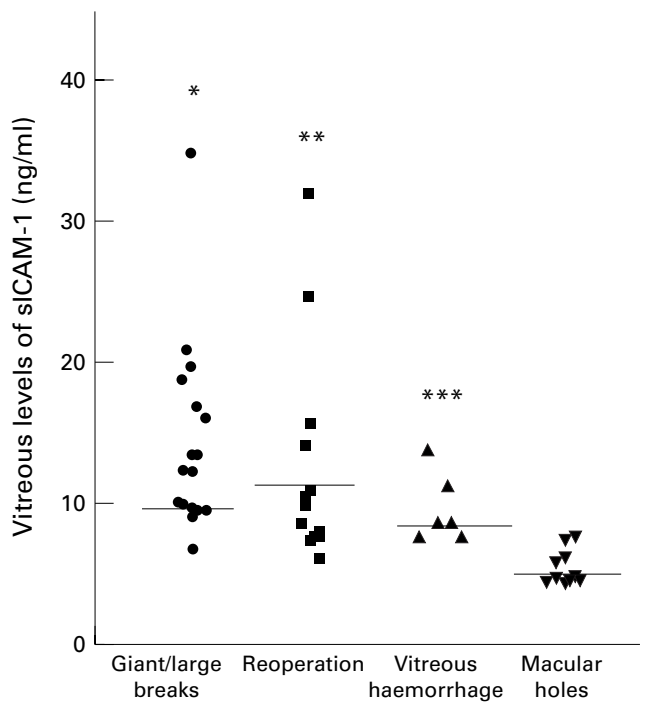

Figure 2 Comparison between vitreous levels of SICAM-1 in eyes with various factors known to predispose to PVR. ${ }^{\star}$ Mann-Whitney U test, $p=0.0003$ (v eyes with macular holes); ${ }^{\star *}$ Mann-Whitney $U$ test, $p=0.00033$ (v eyes with macular holes), $p=0.044$ ( $v$ eyes with vitreous

haemorrhage); ${ }^{\star *}$ Mann-Whitney $U$ test, $p=0.0028$ (v eyes with macular holes).

Whitney U test: $\mathrm{p}=0.00033$ ), and eyes presenting with severe vitreous haemorrhages (range 7.46-13.53; Mann-Whitney U test: $\mathrm{p}=0.0028$ ) when compared with eyes with macular holes (Fig 2). There were no significant differences in sICAM-1 vitreous levels between eyes with large retinal breaks and eyes that had undergone previous operations or that presented with vitreous haemorrhage $(p>0.5)$. However, vitreous from eyes which had been subjected to previous operations contained higher levels of sICAM-1 than eyes with severe vitreous haemorrhage (Mann-Whitney $\mathrm{U}$ test: $\mathrm{p}=0.044)$.

\section{Discussion}

This study shows that vitreous from eyes with RRD at high risk of developing PVR contained higher levels of sICAM than those from eyes with RRD without clinically recognisable risk of PVR. Analysis of the various clinical factors known to predispose to PVR showed that there were no differences in the vitreous levels of sICAM between eyes with large retinal breaks and those with vitreous haemorrhage or history of previous retinal surgery. However, vitreous sICAM-1 levels in eyes with vitreous haemorrhage were lower than in eyes that had undergone previous operations.

Vascular endothelial cells express basal levels of ICAM-1, which are markedly increased during the inflammatory process due to cell activation by cytokines. ${ }^{10}$ Enhanced expression of ICAM-1 is accompanied by membrane shedding of this molecule into surrounding tissues and fluids, ${ }^{15}$ which may explain the increased presence of this molecule in vitreous from eyes with RRD and PVR. That vitreous levels of sICAM-1 are higher in eyes with PVR than in eyes with RRD at high risk of PVR, and that eyes with RRD at high risk of PVR exhibit higher vitreous levels of sICAM-1 than eyes with RRD without clinical risk of PVR, 
reinforces our view that levels of vitreous sICAM-1 depend upon the severity of cytokine mediated reactions at the blood-retinal barrier and therefore upon the severity of inflammation caused by the trauma of RRD.

Since considerable levels of sICAM-1 may be found in normal serum $(102-450 \mathrm{ng} / \mathrm{ml}),{ }^{15}$ it was important to consider the possibility that sICAM-1 found in eyes with vitreous haemorrhage may have derived from blood. As such, one should expect much higher levels of this molecule in vitreous from eyes with vitreous haemorrhage when compared with other clinical risks for PVR. However, sICAM-1 levels in eyes with vitreous haemorrhage were significantly lower than in vitreous from eyes which had undergone previous retinal surgery, strongly suggesting that this molecule is locally released by cells of the retinal microenvironment. This is supported by our previous observations that ICAM-1 expression is increased on the vascular endothelium of retinal vessels during anterior $\mathrm{PVR}^{14}$ and on the extracellular matrix and infiltrating cells of PVR membranes. ${ }^{13}$

Vitreoretinal surgeons would prefer to prevent PVR as the surgery for PVR itself is unpredictable, both anatomically and visually. ${ }^{14}$ The clinical factors known to predispose RRD to PVR include the presence of vitreous haemorrhages, large retinal breaks, longstanding retinal detachments, and previous operations. ${ }^{1920}$ However, at present there are no laboratory tests that may help to identify those at risk of PVR. The present study showed that vitreous levels of sICAM-1 $\geqslant 15 \mathrm{ng} / \mathrm{ml}$ (3x median of the levels present in control eyes) provide a useful cut off point for a highly specific test $(96.7 \%)$ with high positive $(91.6 \%)$ and negative $(96.7 \%)$ predictive values, despite a relatively low sensitivity $(30.5 \%)$. Hence, we propose that assessment of the vitreous levels of sICAM-1 may constitute an additional factor for identifying patients at high risk of developing PVR.

In the present study only one of the 36 patients with high clinical risk factors for PVR developed this complication, which is in accordance with the known incidence of PVR. ${ }^{1}$ On this basis we could not predict PVR development by high vitreous levels of sICAM-1 for which, in our view, the significance of high vitreous levels of sICAM-1 as a risk indicator of PVR lies in the relation between the laboratory findings and the presence of clinical risk factors for PVR.

A common feature of the clinical risk factors of PVR is a high degree of inflammation, which bears the common features of cell migration and release of cytokines and growth factors within the vitreous cavity. ${ }^{6723}$ High vitreous levels of sICAM-1 will raise the index of suspi- cion for the development of PVR, encourage the advisability of monitoring patients after surgery, and the targeting of those likely to benefit from adjunct anti-inflammatory therapy. Laboratory evidence of those at risk of developing PVR will advance the clinical and surgical management of this condition.

We thank Mr R D Hollifield for technical assistance and Miss C Bunce from the Glaxo Institute of Epidemiology at Moorfields Eye Hospital for her help with the statistical analysis.

Supported by The Thomas Pocklington Trust and The Guide Dogs for the Blind Association.

1 Charteris DG. Proliferative vitreoretinopathy: pathobiology, urgical management, and adjunctive treatment. $\mathrm{Br} \mathcal{F} \mathrm{Oph}$ halmol 1995; 79:953-60.

2 Machemer R. Proliferative vitreoretinopathy (PVR): a personal account of its patogenesis and treatment. Invest Ophthalmol Vis Sci 1988;29:1771-83.

3 Wiedemann P, Lemmen K, Schmiedl R, et al. Intraocular daunorubicin for the treatment of prophylaxis in trumatic proliferative vitreoretinopathy. Am f Ophthalmol 1987;104: $10-14$.

4 Ryan S. Traction retinal detachment. XLIX Edward Jackson Memorial Lecture. Am f Ophthalmol 1993;15:1-20.

5 Stern WH, Lewis GP, Erickson PA, et al. Fluorouracil therapy for proliferative vitreoretinopathy. Am $\mathcal{f}$ Ophthalmol therapy for prolifer

6 Limb GA, Little BC, Meager A, et al. Cytokines in proliferative vitreoretinopathy. Eye 1991;5:686-93.

7 Cassidy L, Barry P, Shaw C, et al. Platelet derived growth factor and fibroblast growth factor basic levels in the vitreous of patients with vitreoretinal disorders. Br F Ophthalmol 1998;82:181-5

8 Limb GA, Alam A, Earley O, et al. Distribution of cytokine proteins within epiretinal membranes in proliferative vitreoretinopathy. Curr Eye Res 1994;13:791-8.

9 Vinores SA, Henderer JD, Mahlow J, et al. Isoforms of platelet-derived growth factor and its receptors in epiretinal membranes - immunolocalization to retinal pigmented epithelial cells. Exp Eye Res 1995;60:607-19.

10 Mackay CR, Imhof BA. Cell adhesion in the immune system. Immunol Today 1993;14:99-102.

11 Leeuwenberg JFM, Smeets EF, Neefjes JJ, et al. E-selectin and intercellular adhesion molecule- 1 are released by activated human endothelial cells in vitro. Immunology 1992;77:543-9.

12 Elner SG, Elner VM, Pavilack MA, et al. Modulation and function of intercellular adhesion molecule-1 (CD54) on human retinal pigment epithelial cells. Lab Invest 1992;66: 200-11.

13 Limb GA, Franks WA, Munasinghe KR, et al. Proliferative vitreoretinopathy: an examination of the involvement of lymphocytes, adhesion molecules and HLA-DR antigens. Graefes Arch Clin Exp Ophthalmol 1993;231:331-6.

$14 \mathrm{Limb} \mathrm{GA}$, Chignell AH, Woon $\mathrm{H}$, et al. Evidence of chronic inflammation in anterior retina excised after relaxing retinotomy. Graefes Arch Clin Exp Ophthalmol 1996;234:213-20.

15 Gearing AJH, Newman W. Circulating adhesion molecules in disease. Immunol Today 1993;14:506-12.

16 Zaman AG, Edelsten C, Stanford MR, et al. Soluble intercellular adhesion molecule-1 (sICAM-1) as a marker of disease relapse in idiopathic uveoretinitis. Clin Exp Immunol 1994;95:60-5.

17 Limb GA, Chignell AH, Cole DJ, et al. Vitreous levels of intercellular adhesion molecule-1 (ICAM-1) in proliferative vitreoretinopathy. Invest Ophthalmol Vis Sci 1997;38: 1043-8.

18 Esser P, Bresgen M, Fischbach R, et al. Intercellular adhesion molecule- 1 levels in plasma and vitreous from patients with vitreoretinal disorders. Ger $\mathcal{f}$ Ophthalmol 1995;4:269-74

19 Cowley M, Conway BP, Campochiaro PA, et al. Clinical risk factors for proliferative vitreoretinopathy. Arch Ophthalmol 1989;107:1147-51.

20 Nagasaki H, Shinagawa K. Risk factors for proliferative vitreoretinopathy. Curr Opin Immunol 1995;111:70-5.

21 Machemer R, Aaberg TM, Freeman HM, et al. An updated classification of retinal detachment with proliferative vitreclassification of retinal detachment with proliferative

22 Dunn G, Everitt B. Clinical biostatistics. London: Edward Arnold, 1995:11-33.

23 Yang CH, Cousins S. Quantitative assessment of growth stimulating activity of the vitreous during PVR. Invest Ophthalmol Vis Sci 1992;33:2436-42. 